MITSUBISHI ELECTRIC RESEARCH LABORATORIES

http://www.merl.com

\title{
Non-coherent ToA Estimation for UWB Multipath Channels using Max-eigenvalue Detection
}

Shi, W.; Annavajjala, R.; Orlik, P.V.; Molisch, A.F.; Ochiari, M.; Taira, A.

TR2012-046 June 2012

\begin{abstract}
Due to the fine delay resolution in ultra-wideband (UWB) wireless propagation channels, a large number of multipath components (MPC) can be resolved; and the first arriving MPC might not be the strongest one. This makes time-of-arrival (ToA) estimation, which essentially depends on determining the arrival time of the first MPC, highly challenging. In this paper, we consider noncoherent ToA estimation given a number of measurement trials, at moderate sampling rate and in the absence of knowledge of pulse shape. The proposed ToA estimation is based on detecting the presence of a signal in a moving time delay window, by using the largest eigenvalue of the sample covariance matrix of the signal in the window as the test statistic. We show that energy detection can be viewed as a special case of the eigenvalue detection. Maxeigenvalue detection (MED) generally has superior performance, due to the following reasons: 1) MED collects less noise, namely only the noise contained in the signal space, and 2) if multiple channel taps fall into the time window, the MED detector can collect energy from all of them. Simulation results confirm that MED outperforms the energy detection in IEEE 802.15.3a and 802.15.4a channels. Finally, the selection of the threshold of the MED is studied both by simulations and by random matrix theory.
\end{abstract}

IEEE International Conference on Communications

This work may not be copied or reproduced in whole or in part for any commercial purpose. Permission to copy in whole or in part without payment of fee is granted for nonprofit educational and research purposes provided that all such whole or partial copies include the following: a notice that such copying is by permission of Mitsubishi Electric Research Laboratories, Inc.; an acknowledgment of the authors and individual contributions to the work; and all applicable portions of the copyright notice. Copying, reproduction, or republishing for any other purpose shall require a license with payment of fee to Mitsubishi Electric Research Laboratories, Inc. All rights reserved.

Copyright (C) Mitsubishi Electric Research Laboratories, Inc., 2012

201 Broadway, Cambridge, Massachusetts 02139 



\title{
Non-coherent ToA Estimation for UWB Multipath Channels using Max-eigenvalue Detection
}

\author{
Wei Shi ${ }^{1}$, Ramesh Annavajjala ${ }^{2}$, Philip V. Orlik ${ }^{2}$, Andreas F. Molisch ${ }^{3}$, Mari Ochiai ${ }^{4}$, and Akinori Taira ${ }^{4}$ \\ ${ }^{1}$ Dept. of Electrical Engineering, Univ. of Washington, Seattle, WA, 98195 \\ ${ }^{2}$ Mitsubishi Electric Research Labs, 201 Broadway, Cambridge, MA, 02139 \\ ${ }^{3}$ Dept. of Electrical Engineering, Univ. of Southern California, Los Angeles, CA, 90089 \\ ${ }^{4}$ Mitsubishi Electric Corp., 5-1-1 Ofuna, Kamakura, Kanagawa 247-8501, Japan
}

\begin{abstract}
Due to the fine delay resolution in ultra-wideband (UWB) wireless propagation channels, a large number of multipath components (MPC) can be resolved; and the first arriving MPC might not be the strongest one. This makes time-of-arrival (ToA) estimation, which essentially depends on determining the arrival time of the first MPC, highly challenging. In this paper, we consider non-coherent ToA estimation given a number of measurement trials, at moderate sampling rate and in the absence of knowledge of pulse shape. The proposed ToA estimation is based on detecting the presence of a signal in a moving time delay window, by using the largest eigenvalue of the sample covariance matrix of the signal in the window as the test statistic. We show that energy detection can be viewed as a special case of the eigenvalue detection. Maxeigenvalue detection (MED) generally has superior performance, due to the following reasons: 1) MED collects less noise, namely only the noise contained in the signal space, and 2) if multiple channel taps fall into the time window, the MED detector can collect energy from all of them. Simulation results confirm that MED outperforms the energy detection in IEEE 802.15.3a and 802.15.4a channels. Finally, the selection of the threshold of the MED is studied both by simulations and by random matrix theory.
\end{abstract}

\section{INTRODUCTION}

There is a growing demand for accurate location estimation or positioning, which is a key functionality for location-aware systems. Wireless radio frequency localization is of significant interest especially in cluttered indoor environments or underground scenarios where the global positioning system (GPS) is not suitable. Occupying large bandwidth, ultra-wideband (UWB) signalling offers great potential for high accuracy positioning, due to its ability to resolve multipath components (MPCs) and penetrate obstacles [4] [6]. Most UWB localization systems are based on range measurements between nodes, obtained from estimating the Time of Arrival (ToA) of the first path of the received ranging signal [6]. Ranging is based on the assumption that the ToA of the first detected MPC equals the geometrical distance between transmitter and receiver, divided by the speed of light. Thus, the accuracy of the ToA estimation is affected not only by noise, but also multipath propagation, and the absence of true line-of-sight (LOS). In dense multipath channels without LOS, the first detected path is often not the strongest, and sometimes considerably weaker than later MPCs, thus making its identification challenging [9].

This work was conceived and completed during the first author's internship at MERL.
Considerable research has been done on ToA estimation in multipath environments that is closely related to traditional channel estimation where channel amplitudes and delays are jointly estimated. Examples include the maximum likelihood method (ML) [3] and a generalized ML ratio test [4]. However, such estimators require fast sampling (at least at the Nyquist rate), which increases the required hardware effort and energy consumption, and suffer from high computational complexity due to large number of multipath components. Furthermore, they do not necessarily perform better than simpler threshold-based estimators (see [9] and reference therein).

A non-coherent and low-complexity alternative is the threshold-based energy detector (ED) [8] [9]. The ED detects the signal by comparing the received signal energy to a threshold. The main advantages of the ED are possibility for sub-Nyquist sampling rate, the potential for analog implementation, and no requirements on the knowledge of the signal shape. Another threshold-based detector is the classical correlation (or matched filter) estimator. The received signal is correlated with the transmitted signal template and then the peak of the correlator output is detected as the estimated ToA. The correlation estimator achieves the Cramer-Rao lower bound (CRLB) at high signal-tonoise-ratios (SNRs). However, the correlation method requires knowledge of the arriving pulse shape and fine clock timing. A dense multipath channel and frequency-selective propagation effects [13] make the received pulse templates different in shape from the transmitted ones, and thus degrade the performance of the correlation estimator.

In this paper, we propose a new threshold-based estimator for the non-coherent ToA estimation problem given a number of measurement trials, a moderate sampling rate and absence of knowledge of the pulse shape. The proposed ToA estimator is based on the largest eigenvalue of the covariance matrix of the received signal in a moving window. Operating at a few times (e.g., 5 times) the sampling rate of $\mathrm{ED}$, the max-eigenvalue detector (MED) exhibits significant performance improvement over ED. The superior performance of MED is explained by that the largest eigenvalue contains less noise energy and higher signal energy is collected from multiple channel taps. Compared with ED that collects the noise energy in all the subspaces, MED only collects the noise energy contained in the signal subspace. The better energy collection comes from a longer window to cover several adjacent MPCs while the noise energy collected remains unchanged as the window size changes. As a further 
contribution, this paper presents the threshold of MED for a given false alarm rate, based on random matrix theory. Finally, we show that MED is a generalization of ED since it includes the latter as a special case. Many advanced techniques originally designed for ED, such as the leading edge search algorithms [7], can also apply to MED with little modification.

It is worth noting the differences between MED and the socalled super-resolution ToA estimation [12]. The super-resolution approach takes the Fourier transform of the time-domain signal, and applies, e.g., the multiple signal classification (MUSIC) algorithm to resolve MPCs. First, while MUSIC and similar algorithms intend to use the super-resolution array algorithm to resolve MPCs from each other, our work focuses on improving the ability to detect the presence of either one channel path or a cluster of multiple channel taps. Indeed, our approach works particularly well in dense multipath environments in which energy from adjacent channel paths are close enough to be collected in one window. Secondly, the MUSIC algorithm requires accurate estimation of the number of signals, which is difficult when some MPCs are weak or there exists a large number of MPCs. Our work, on the other hand, simply determines whether a signal exists in a certain window or not and thus does not require such order estimation. Finally, the MUSIC algorithm needs to know the pulse shape, which may be impractical for the reasons stated in the previous paragraph.

\section{The ToA Estimation}

\section{A. Multipath Channels}

The impulse response of a UWB multipath channel can be written as [13]

$$
h(t)=\sum_{l=1}^{L} \alpha_{l} \xi_{l}\left(t-\tau_{l}\right),
$$

where $L$ is the number of multipath components, $\left\{a_{1}, \cdots, a_{L}\right\}$ and $\left\{\tau_{1}, \cdots, \tau_{L}\right\}$ are the sets of the channel gains and path delays, respectively, the $\xi_{l}(\tau)$ are the pulse distortion functions due to frequency selectivity of the propagation effects, and $\tau_{1}$ is the ToA to be estimated. ${ }^{1}$ Let the transmitter send a pulse $\sqrt{E} p(t)$ where $E$ is the pulse energy and $p(t)$ is a unit-energy pulse shape, Assuming the transmitter and the receiver are synchronized, the received signal can be written as

$$
r(t)=s(t)+n(t)=\sqrt{E} \sum_{l=1}^{L} \alpha_{l} p\left(t-\tau_{l}\right)+n(t),
$$

where $s(t)$ denotes the signal after distortion by the multipath channels, $n(t)$ is additive white Gaussian noise with mean zero and two-sided power spectral density $N_{0} / 2$.

The ToA estimation is based either on the received signal resulting from a single transmitted pulse or a number of consecutive pulses. A large number of pulses can be transmitted within one coherence time of the channel (i.e., during a time over which the channel stays essentially constant: the typical channel coherence time for a UWB channel is of order of tens

\footnotetext{
${ }^{1}$ by this formulation, we assume that the runtime of the first MPC is a measure of the distance between transmitter and receiver. If a direct component is completely blocked, and/or slowed by being transmitted through a dielectric medium, the ToA of the first component will not correspond to the geometric distance. This effect, while important, is beyond the scope of the paper and will not be considered further here.
}

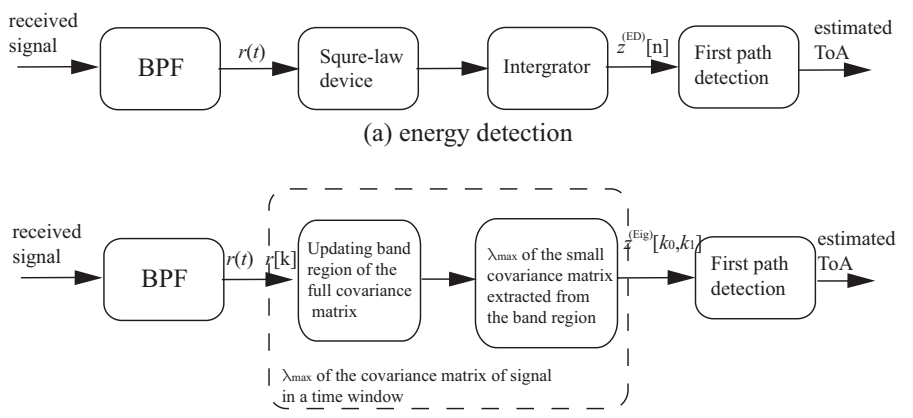

(b) max-eigenvalue detection

Fig. 1. Block diagram of (a) the energy detection based ToA estimator and (b) the max-eigenvalue based ToA estimator.

of milliseconds (ms) [14], while the largest channel delay for the weakest multipath component is about 100 nanoseconds (ns), observed from channel realizations of UWB channel models [1], [2]. This means that the total transmission time for 500 pulses is only $0.05 \mathrm{~ms}$, which is much smaller than the coherence time. However, due to the lack of a phase-locked loop in the noncoherent UWB receiver, the received signals from different trial is assumed to have independent unknown phases, that is,

$$
r_{j}(t)=e^{i \varphi_{j}} \sqrt{E} \sum_{l=1}^{L} \alpha_{l} p\left(t-\tau_{l}\right)+n_{j}(t), j=1, \cdots, M
$$

where $j$ is the trial index, $M$ is the number of trials, and $\left\{\varphi_{j}\right\}_{j=1}^{M}$ are unknown phases. Both ED and MED are immune to the unknown phases, as seen later.

\section{B. Energy Detection (ED)}

ED is a low-complexity detection method that does not require knowledge of the pulse shape and operates at symbol-time samples. As shown in Fig. 1 (a), the received signal is first passed through a bandpass filter to eliminate out-of-band noise, then a square-law device and an integrator to collect energy within a chosen time window. The integrator output samples can be expressed as

$$
z^{(\mathrm{ED})}[n]=\frac{1}{M} \sum_{j=1}^{M} \int_{(n-1) T_{b}}^{n T_{b}}\left|r_{j}(t)\right|^{2} \mathrm{~d} t,
$$

where $n$ denotes the sample index, $T_{b}$ denotes the integration length, and the output samples are averaged over $M$ trials to reduce the noise contribution. For later comparison with MED, let $T_{s}$ denote the sampling period of MED and assume $T_{b}=$ $W^{(\mathrm{ED})} T_{s}$. We call $W^{(\mathrm{ED})}$ the window size of ED.

The ToA is then estimated based on the energy samples. One of the low-complexity first path detection techniques is the threshold-based estimation. In the threshold-based approaches, each energy sample $z^{(\mathrm{ED})}[n]$ is compared with a threshold to decide whether signal is present or not. If $z^{(\mathrm{ED})}[n]>\gamma^{(\mathrm{ED})} \delta_{n}^{2}$ where $\delta_{n}^{2}$ is the noise variance and $\gamma^{(\mathrm{ED})}$ is a threshold, signal presence is assumed. Note that the maximum energy sample may not be the true ToA in multipath condition. This motivates many leading edge search algorithms such as largest- $N$ peak detection, jump-back-and-search-forward and serial-backward-search etc. (see [6], [7] and the references therein). In this paper, we only consider a simple leading edge detection that simply chooses the earliest larger-than-threshold sample as the estimated ToA. 


\section{Max-eigenvalue Detection (MED)}

In this subsection, we first discuss the preliminaries on the max-eigenvalue method for array signal detection. Then we propose a new ToA estimation method by casting signal detection within a time window as an array signal detection problem.

The maximum eigenvalue can be used as a test statistic for array signal detection [5]. There are two hypotheses for the received array signal:

$$
\begin{aligned}
& \mathcal{H}_{0}: \mathbf{y}=\mathbf{n}, \\
& \mathcal{H}_{1}: \mathbf{y}=\mathbf{s}+\mathbf{n},
\end{aligned}
$$

where $\mathbf{y}$ denotes the received array signal, $\mathbf{n} \sim \mathcal{N}\left(\mathbf{0}, \sigma_{n}^{2} \mathbf{I}\right)$ represents real-valued Gaussian noise or $\mathbf{n} \sim \mathcal{C N}\left(\mathbf{0}, \sigma_{n}^{2} \mathbf{I}\right)$ complexvalued Gaussian noise, and $\mathbf{s}$ is the received source signal. Note that $\mathbf{s}$ can be either $\mathbf{s}=\mathbf{a} s$ representing one source signal $s$ associated with a steering vector $\mathbf{a}$, or $\mathbf{s}=\mathbf{a}_{1} s_{1}+\mathbf{a}_{2} s_{2}+\cdots$ representing superposition of multiple sources.

The statistical covariance matrix of the received signal is defined as $\mathbf{R}_{\mathbf{y}}=E\left\{\mathbf{y} \mathbf{y}^{H}\right\}$, and it can be verified that under the two hypotheses $\mathbf{R}_{\mathbf{y}}$ is

$$
\begin{array}{ll}
\mathbf{R}_{\mathbf{y}}=\sigma_{n}^{2} \mathbf{I}, & \text { for } \mathcal{H}_{0}, \\
\mathbf{R}_{\mathbf{y}}=\mathbf{R}_{\mathbf{s}}+\sigma_{n}^{2} \mathbf{I}, & \text { for } \mathcal{H}_{1},
\end{array}
$$

where $\mathbf{R}_{\mathbf{s}}=E\left\{\mathbf{s s}^{H}\right\}$. Since the above equations apply to both the complex and the real-valued case, redundant discussions for the real-valued case are omitted. The largest eigenvalues of $\mathbf{R}$ under the two hypotheses are

$$
\begin{array}{ll}
\lambda_{\max }\left(\mathbf{R}_{\mathbf{y}}\right)=\sigma_{n}^{2}, & \text { for } \mathcal{H}_{0}, \\
\lambda_{\max }\left(\mathbf{R}_{\mathbf{y}}\right)=\lambda_{\max }\left(\mathbf{R}_{s}\right)+\sigma_{n}^{2}, & \text { for } \mathcal{H}_{1},
\end{array}
$$

Thus $\lambda_{\max }\left(\mathbf{R}_{\mathbf{y}}\right)$ can be used to detect the presence of a signal. If the signal $\mathbf{s}$ is not present, then $\lambda_{\max }\left(\mathbf{R}_{\mathbf{y}}\right)=\sigma_{n}^{2}$. If the signal exists, then $\lambda_{\max }\left(\mathbf{R}_{\mathbf{y}}\right)>\sigma_{n}^{2}$.

In practice, the decision of array signal detection is made after observing a number of snapshots $\left\{\mathbf{y}_{1}, \cdots, \mathbf{y}_{M}\right\}$ where $\mathbf{y}_{j}$ denotes the received signal at the $j$-th snapshot and $M$ is the number of snapshots. Because $M$ is limited, a perfect statistical covariance matrix is not available. The statistical covariance matrix can be approximated by the sample covariance matrix, defined as $\hat{\mathbf{R}}_{\mathbf{y}}=\frac{1}{M} \sum_{j=1}^{M} \mathbf{y}_{j} \mathbf{y}_{j}^{H}$. The sample covariance matrix will approach the statistical covariance matrix as $M$ increases. Moreover, note that the sample covariance matrix is Hermitian and thus only upper or lower triangular elements need to be calculated.

Next we return to the ToA estimation problem given the received signal $\left\{r_{1}(t), \cdots, r_{M}(t)\right\}$ from the $M$ trials. The received signal is sampled at a moderately high sampling rate. Let

$$
r_{j}[k]=r_{j}\left(k T_{s}\right)=s_{j}\left(k T_{s}\right)+n_{j}\left(k T_{s}\right), j=1, \cdots, M
$$

denote the discrete-time samples of the $j$-th trial, where $k$ denotes index and $T_{s}$ denotes the sampling period, and let $\sigma_{n}^{2}$ denote the variance of the noise sample $n_{j}\left(k T_{s}\right)$. Consider the signal samples within a moving time window $\left[k_{0} T_{s}, k_{1} T_{s}\right]$, where the window size is defined as $W^{(\mathrm{Eig})}=\left(k_{1}-k_{0}+1\right)$. From (3), the windowed samples at the $j$-th trial can be written as ${ }^{2}$

$$
\begin{gathered}
\mathbf{r}_{j}\left[k_{0}, k_{1}\right]=\left[\begin{array}{c}
r_{j}\left[k_{0}\right] \\
\vdots \\
r_{j}\left[k_{1}\right]
\end{array}\right]=\left[\begin{array}{c}
r_{j}\left(k_{0} T_{s}\right) \\
\vdots \\
r_{j}\left(k_{1} T_{s}\right)
\end{array}\right] \\
=e^{i \varphi_{j}} \sqrt{E}\left[\mathbf{p}_{1}, \cdots, \mathbf{p}_{L}\right]\left[\begin{array}{c}
\alpha_{1} \\
\vdots \\
\alpha_{L}
\end{array}\right]+\left[\begin{array}{c}
n_{j}\left(k_{0} T_{s}\right) \\
\vdots \\
n_{j}\left(k_{1} T_{s}\right)
\end{array}\right]
\end{gathered}
$$

where

$$
\mathbf{p}_{l}=\left[\begin{array}{c}
p\left(k_{0} T_{s}-\tau_{l}\right) \\
\vdots \\
p\left(k_{1} T_{s}-\tau_{l}\right)
\end{array}\right]
$$

is the pulse shape for the $l$-th channel tap appearing in the window. Because the pulse has limited duration, (9) can be reduced into two hypotheses: $\mathcal{H}_{0}$ means all channel taps are far away from the window and thus no signal appears in the window, and $\mathcal{H}_{1}$ there exists at least one channel tap whose distance to the window is less than the pulse duration.

$$
\begin{aligned}
& \mathcal{H}_{0}: \mathbf{r}\left[k_{0}, k_{1}\right]=\mathbf{n}, \\
& \mathcal{H}_{1}: \mathbf{r}\left[k_{0}, k_{1}\right]=\mathbf{s}+\mathbf{n} .
\end{aligned}
$$

After observing $M$ trials, the sample covariance matrix for a window $\left[k_{0} T_{s}, k_{1} T_{s}\right]$ can be estimated by

$$
\hat{\mathbf{R}}\left[k_{0}, k_{1}\right]=\frac{1}{M} \sum_{j=1}^{M} \mathbf{r}_{j}\left[k_{0}, k_{1}\right]\left(\mathbf{r}_{j}\left[k_{0}, k_{1}\right]\right)^{H},
$$

and its largest eigenvalue can be used to detect whether there exists a channel tap near the window. As the window moves, the sequence of the largest eigenvalues is

$$
z^{(\mathrm{Eig})}\left[k_{0}, k_{1}\right]=\lambda_{\max }\left(\hat{\mathbf{R}}\left[k_{0}, k_{1}\right]\right),
$$

which will be compared with a threshold $\gamma^{(\text {Eig })} \sigma_{n}^{2}$. The leadingedge window is the earliest one that detects the presence of signal. The end time $k_{1} T_{s}$ of the leading-edge window is chosen as the estimated ToA. An example of the ToA estimation is given in Fig. 2. Furthermore, note that the advanced leading edge search algorithms originally designed for the energy samples $z^{(\mathrm{ED})}$ can also apply to the max-eigenvalue sequence. Also it is possible to make more accurate adjustments, such as considering several consecutive windows with positive detection outcomes, or further refining within one window. These further techniques are not discussed here for not being the main focus of our paper.

\section{IMPLEMENTATION OF THE MED}

\section{A. Reducing Duplicate Calculation of the Sample Covariance Matrices}

In calculating the sample covariance matrices of adjacent windows, it can be seen from (12) that $\hat{\mathbf{R}}\left[k_{0}, k_{1}\right]$ and $\hat{\mathbf{R}}\left[k_{0}+1, k_{1}+1\right]$ have a large number of common elements. Thus it is desired to reduce the computational load by avoiding duplicate calculation. In fact, if we define a full covariance matrix $\hat{\mathbf{R}}$ as

$$
\hat{\mathbf{R}}[0, K]=\frac{1}{M} \sum_{j=1}^{M} \mathbf{r}_{j} \mathbf{r}_{j}^{H},
$$

\footnotetext{
${ }^{2}$ This model of the received signal assumes that the phase drift of the receiver is negligible for the duration of the time window.
} 
where $\mathbf{r}_{j}=\left[r_{j}(0), r_{j}\left(T_{s}\right), \cdots, r_{j}\left(K T_{s}\right)\right]^{T}$ denotes the full samples and $\left[0, K T_{s}\right]$ is the total observation time, then $\hat{\mathbf{R}}\left[k_{0}, k_{1}\right]$ will be a sub-matrix of $\hat{\mathbf{R}}$. When moving the time window, a series of $\hat{\mathbf{R}}\left[k_{0}, k_{1}\right]$ covers a band region of $\hat{\mathbf{R}}$. The bandwidth ${ }^{3}$ is equal to two times the window size minus one. Also note that only the diagonal elements plus half band needs to be calculated, because $\hat{\mathbf{R}}$ is a Hermitian matrix. As a result, we replace the calculation of a series of $\hat{\mathbf{R}}\left[k_{0}, k_{1}\right]$ (12) by two stages:

1) Calculate half of the band region of $\hat{\mathbf{R}}[0, K]$ using that the $i, j$-th elements of $\hat{\mathbf{R}}[0, K]$ is given by $\frac{1}{M} \sum_{j=1}^{M} r_{j}\left((i-1) T_{s}\right) r_{j}^{*}\left((j-1) T_{s}\right)$. Complete the other half by conjugate transpose.

2) Extract the covariance matrix $\hat{\mathbf{R}}\left[k_{0}, k_{1}\right]$ from the band region and calculate (13).

\section{B. Threshold Selection for Max-eigenvalue Detection}

The selection of the threshold carries critical importance for accurate ToA estimation. For different channel models and at different SNRs, an optimal threshold can be selected to minimize the average estimation error. The evaluation of the average error is usually done by numerical simulation or experiments.

An alternative way to set the threshold is to choose a threshold to achieve a certain false alarm rate for the noise-only time window. From random matrix theory, the distribution of the largest eigenvalue of a real-valued (complex-valued) noise-only Wishart matrix approaches a Tracy-Widom distribution of order 1 (order 2) as both the dimensions go to infinity [11]. In ref. [11] it is reported that for a moderate number of dimensions this distribution also fits the actual distribution.

Real-valued case [11]: Denote the Wishart matrix $\mathbf{A}_{1}=\mathbf{X X}^{T}$ where $\mathbf{X}=\left(\mathbf{X}_{i, j}\right)_{W \times M}$ has i.i.d. entries $X_{i, j} \sim \mathcal{N}(0,1)$. The distribution of $\frac{\lambda_{\max }\left(\mathbf{A}_{1}\right)-\mu_{1}}{v_{1}}$ where

$$
\begin{aligned}
& \mu_{1}=(\sqrt{M-1}+\sqrt{W})^{2} \\
& v_{1}=(\sqrt{M-1}+\sqrt{W})\left(\frac{1}{\sqrt{M-1}}+\frac{1}{\sqrt{W}}\right)^{1 / 3}
\end{aligned}
$$

approaches a Tracy-Widom distribution of order 1 (TW1), whose cumulative distribution function (CDF) is given by

$$
F_{1}(s)=\exp \left\{-\frac{1}{2} \int_{s}^{\infty} q(x)+(x-s) q^{2}(s) \mathrm{d} x\right\}
$$

where $q$ solves the non-linear Painlevvé II differential equation

$$
q^{\prime \prime}(x)=x q(x)+2 q^{3}(x) .
$$

When the number of trials is $M$ and the window size is $W$, the noise-only sample covariance is given by $\hat{\mathbf{R}}_{n}=$ $\frac{1}{M} \sum_{j=1}^{M} \mathbf{n}_{j} \mathbf{n}_{j}^{T}=\frac{M}{\sigma_{n}^{2}} \mathbf{A}_{1}$ where $\mathbf{n}_{j} \sim \mathcal{N}\left(0, \sigma_{n}^{2} \mathbf{I}\right)$. For the MED to have a false alarm rate $P_{f a}^{(\mathrm{Eig})}$, the threshold should satisfy

$$
\begin{aligned}
P_{f a}^{(\mathrm{Eig})} & =\operatorname{Pr}\left\{\lambda_{\max }\left(\hat{\mathbf{R}}_{n}\right)>\gamma_{1}^{(\mathrm{Eig})} \sigma_{n}^{2}\right\} \\
& =\operatorname{Pr}\left\{\frac{\sigma_{n}^{2}}{M} \lambda_{\max }\left(\mathbf{A}_{1}\right)>\gamma_{1}^{(\mathrm{Eig})} \sigma_{n}^{2}\right\} \\
& \approx 1-F_{1}\left(\frac{\gamma_{1}^{(\mathrm{Eig})} M-\mu_{1}}{v_{1}}\right) .
\end{aligned}
$$

${ }^{3}$ The bandwidth of a band matrix is defined as the number of diagonals.
This results in the threshold to be

$$
\gamma_{1}^{(\mathrm{Eig})}=\frac{\mu_{1}+v_{1} F_{1}^{-1}\left(1-P_{f a}^{(\mathrm{Eig})}\right)}{M} .
$$

Complex-valued case [11]: For $\mathbf{A}_{2}=\mathbf{X X}^{H}$ where $X_{i, j} \sim$ $\mathcal{C N}(0,1)$, the distribution of $\frac{\lambda_{\max }\left(\mathbf{A}_{2}\right)-\mu_{2}}{v_{2}}$ where

$$
\begin{aligned}
& \mu_{1}=(\sqrt{M}+\sqrt{W})^{2} \\
& v_{1}=(\sqrt{M}+\sqrt{W})\left(\frac{1}{\sqrt{M}}+\frac{1}{\sqrt{L}}\right)^{1 / 3}
\end{aligned}
$$

approaches to Tracy-Widom distribution of order 2 (TW2), whose $\mathrm{CDF}$ is given by

$$
F_{2}(s)=\exp \left\{-\int_{s}^{\infty}(x-s) q^{2}(s) \mathrm{d} x\right\}
$$

where $q$ is still the Painlevé II function defined in (17).

The threshold for the complex case should be set to

$$
\gamma_{2}^{(\mathrm{Eig})}=\frac{\mu_{2}+v_{2} F_{2}^{-1}\left(1-P_{f a}^{(\mathrm{Eig})}\right)}{M} .
$$

It is generally difficult to evaluate $F_{1}, F_{2}$ or $F_{1}^{-1}, F_{2}^{-1}$. Fortunately look-up tables for both $F_{1}$ and $F_{2}$ are already computed off-line [10].

\section{Comparison between ED and MED}

The full description of the detection performance are generally characterized by probability of detection $P_{d}$ and probability of false alarm $P_{f a}$. For the energy detector, the noise-only and the signal-plus-noise energy samples have a centralized and a non-centralized Chi-square distribution, respectively, based on which $P_{f a}^{(\mathrm{ED})}$ and $P_{d}^{(\mathrm{ED})}$ can be expressed in closed form [8]. Aside from these complex closed forms, approximated Gaussian distributions for the energy samples are given in [7], based on which $P_{f a}^{(\mathrm{ED})}$ and $P_{d}^{(\mathrm{ED})}$ can be expressed in simple forms of $Q$-function. However, for the max-eigenvalue detection, the expression of $P_{f a}^{(\mathrm{Eig})}$ is difficult to compute, and the distribution of the largest eigenvalue of signal-plus-noise covariance matrix is unclear so that $P_{d}^{(\mathrm{Eig})}$ remains an open problem. For these reasons, we do not compare in terms of $P_{d}$ and $P_{f a}$, but only compare the means of the test statistics of the two hypotheses.

To facilitate the comparison to MED, we rewrite the integration (4) of ED in terms of discrete samples. Assume that the sampling period $T_{s}$ is high enough, so (4) can be well approximated by

$$
\begin{aligned}
& z^{(\mathrm{ED})}[n]=\frac{1}{M} \sum_{j=1}^{M} \int_{(n-1) T_{b}}^{n T_{b}}\left|r_{j}(t)\right|^{2} \mathrm{~d} t \\
\approx & \frac{T_{s}}{M} \sum_{j=1}^{M}\left\{\begin{array}{l}
\left|r_{j}\left((n-1) T_{b}\right)\right|^{2}+\left|r_{j}\left((n-1) T_{b}+T_{s}\right)\right|^{2} \\
+\cdots+\left|r_{j}\left(n T_{b}\right)\right|^{2}
\end{array}\right\}
\end{aligned}
$$

where $T_{b}=W^{(\mathrm{ED})} T_{s}$ is the window size of the energy detection.

1) For ED, the means of the energy outcomes are

$$
\begin{array}{ll}
E\left\{z^{(\mathrm{ED})}\right\} / T_{s}=W^{(\mathrm{ED})} \sigma_{n}^{2}, & \text { for } \mathcal{H}_{0}, \\
E\left\{z^{(\mathrm{ED})}\right\} / T_{s}=E_{s}+W^{(\mathrm{ED})} \sigma_{n}^{2}, & \text { for } \mathcal{H}_{1},
\end{array}
$$


where $E_{s}$ denotes the energy of the signal within the considered window. For MED, the means of the max-eigenvalue are

$$
\begin{array}{ll}
E\left\{z^{(\mathrm{Eig})}\right\} \approx \sigma_{n}^{2}, & \text { for } \mathcal{H}_{0}, \\
E\left\{z^{(\mathrm{Eig})}\right\} \approx E_{s}+\sigma_{n}^{2}, & \text { for } \mathcal{H}_{1},
\end{array}
$$

where the approximate equalities becomes strict equalities as the number of trials approaches infinity. Comparing (24) with (25), the noise energy gathered by ED is $W^{(\mathrm{ED})}$ times that of MED.

MED only collects energy on the 1-dim subspace (which is the signal subspace) of the eigenvector corresponding to the largest eigenvalue, while ED does not make such distinction. ED collects the total energy distributed in all subspaces. Related to MED, ED uses the sum of all the eigenvalues to detect, i.e.

$$
z^{(\mathrm{ED})}\left[k_{0}, k_{1}\right]=\operatorname{Tr}\left(\hat{\mathbf{R}}\left[k_{0}, k_{1}\right]\right)=\sum \lambda_{i}\left(\hat{\mathbf{R}}\left[k_{0}, k_{1}\right]\right),
$$

where $\operatorname{Tr}(\cdot)$ denotes the trace of a matrix and $\lambda_{i}$ the $i$-th eigenvalue. Thus ED can be interpreted as a special case of MED.

2) For dense multipath channels, several paths arrive successively in the form of a cluster. Each path may have too low energy to be detected individually, but their energy together may be high enough to enable detection. ED has to choose a short window size, usually the same as the pulse duration, since the longer the window size the more noise energy. However, MED can choose a longer window size in order to cover multiple channel taps, as long as the number of trials is large enough to stabilize the eigenvalues. For this reason, MED is able to collect energy from several adjacent channel taps to avoid missing detection of an entire cluster. In the presence of multipath or at low SNR conditions, missing detection, resulting in large errors with magnitudes much larger than the pulse width, dominates the ToA estimation errors. In such harsh conditions, MED outperforms ED by successful detection of a cluster and avoidance of the large errors, although the ToA ambiguity (small errors) within a cluster may not be resolved.

\section{Simulation Results}

This section shows simulations to compare the ToA estimation performance of MED and ED. The simulations are done using channel model CM2 (NLOS 0-4m) of IEEE 802.15.3a [1] and CM2 (residential NLOS 7-20m) of IEEE 802.15.4a [2], which are two of the most comprehensive standardized UWB channel models currently available.

Fig. 2 compares the output of MED and ED. The top subfigure shows a realization of the noisy received signal under the 802.15.3a CM2 channel model, where two channel paths have delays $\tau_{1}=1.1 \mathrm{~ns}$ and $\tau_{2}=3.3 \mathrm{~ns}$, the signal pulse after the multipath channel is plotted red and the noise black. Given $M=1000$ measurements of the received signal, the sequence of the largest eigenvalue and the energy output is calculated and displayed in the middle and lower subfigures, respectively. The 2nd subfigure shows $z^{\text {(Eig) }}\left[k_{0}, k_{0}+W^{(\text {Eig) }}\right]$ where $W^{(\text {Eig) }}=10$ with sampling period $T_{s}=0.1 \mathrm{~ns}$. As the window moves, the max-eig output shows a distinct rise when the window is located at $[0.0,1.0]$, and reaches its maximum when the window is located at $[0.3,1.3]$. If the threshold is properly selected, such as $\lambda_{\max }>1.4 \sigma_{n}^{2}$, then the window $[0.1,1.1]$ is the earliest largerthan-threshold window and the estimated ToA is chosen as the end time $1.1 \mathrm{~ns}$. In the 3 rd subfigure, ED chooses a window size of $T_{b}=3 T_{s}=0.3 \mathrm{~ns}$ to cover the majority of the pulse shape
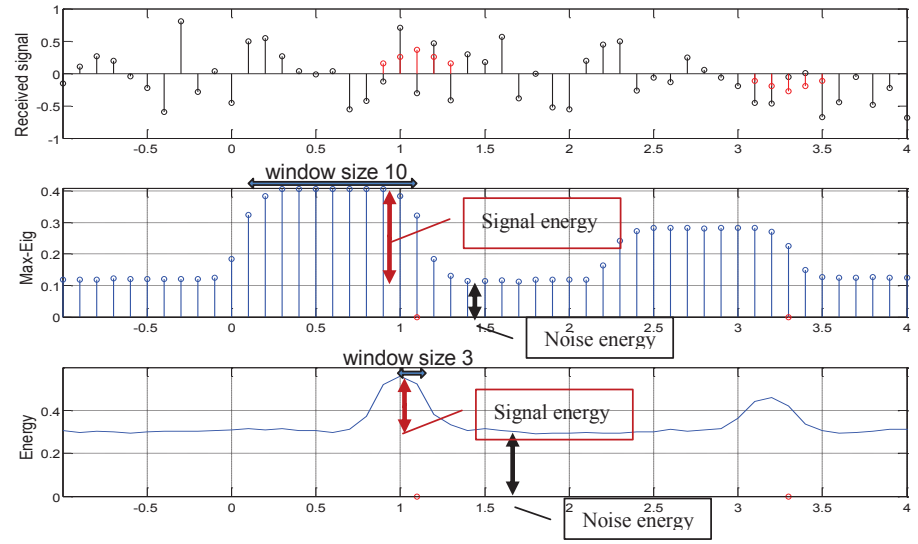

Fig. 2. a) A realization of the received signal under 802.15.3a CM2, where there exist two channel taps at $1.1 \mathrm{~ns}$ and $3.3 \mathrm{~ns}$, the red stems represent the signal after the multipath channel and the black ones noise. b) The max-eigenvalue samples, where the $\mathrm{x}$-axis indicates the start time of the moving window. For a threshold $1.4 \sigma_{n}^{2}$, the earliest larger-than-threshold window is located at $[0.1,1.1]$, and then the ToA is estimated as the end time $1.1 \mathrm{~ns}$. c) The ED output. It is seen that MED has more contrast energy separation between the signal-plus-noise and the noise-only hypotheses.

and in the meanwhile to avoid collecting too much noise energy. As expected from the reasons given in the previous section, the energy separation of MED is more pronounced than that of ED.

Fig. 3 compares the root mean square errors (RMSE) of MED and ED under 802.15.3a CM2, and Fig. 4 under 802.15.4a CM2. The performance of MED is significantly better than that of ED. The cost of MED in terms of the sampling rate is reasonable: the sampling rates are $f_{s}^{(\mathrm{Eig})}=\frac{1}{T_{s}}, f_{s}^{(\mathrm{ED})}=\frac{1}{T_{b}}=\frac{1}{W^{(\mathrm{ED})} T_{s}}$ and $f_{s}^{(\mathrm{Eig})} / f_{s}^{(\mathrm{ED})}=W^{(\mathrm{ED})}=3$.

Finally, we discuss the impact of different window sizes on the ToA estimation accuracy. For ED, the window size obviously changes the noise energy collected by the window. The window $W^{(\mathrm{ED})}$ has to be large enough to be able to cover the major pulse shape, and on the contrary, has to be small in order to avoid too much noise energy. Fig. 5 shows that the choice of window size does impact the estimation accuracy. For MED, the window size can be large to cover multiple channel taps, but not too large for stabilizing the eigenvalues, given limited number of trials. However, the problem of choice of window size for MED is not as critical as the that of $\mathrm{ED}$, as long as the window size is large enough to cover the whole pulse shape. Fig. 6 plots the effect of different window sizes on the estimation accuracy. As seen in the figure, for different window size $W^{(\mathrm{Eig})}=5,8,10$, the minimum achievable RMSEs are almost the same.

\section{CONCLUSION}

We have proposed a threshold-based ToA estimator based on the largest eigenvalue of the covariance matrix of a moving window. The proposed method operates at moderately high sampling rate, does not need the knowledge of the pulse shape and imposes little computational complexity. The proposed maxeigenvalue method only collects the noise energy distributed in the signal subspace, which is the main advantage over the energy-detection method. We have provided a calculation scheme that avoids duplicate calculations for adjacent time window to reduce the computational load. The selection of the threshold is also discussed using random matrix theory. Simulation results 


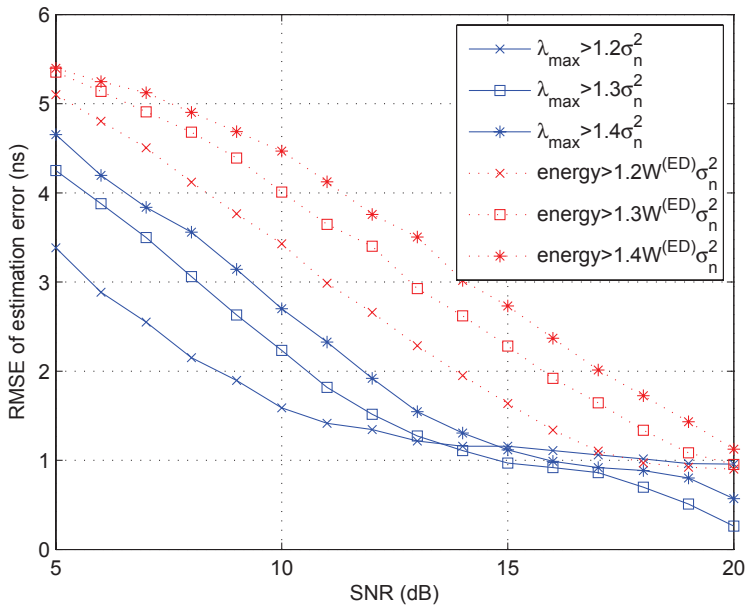

Fig. 3. Comparison of RMSE of ToA estimation errors of MED and ED, under 802.15.3a CM2 and $M=500$ trials. The other parameters for MED are $T_{s}=0.1 \mathrm{~ns}$ and $W^{(\mathrm{Eig})}=5$, and those for ED are $W^{(\mathrm{ED})}=3$ and $T_{b}=W^{(\mathrm{ED})} T_{s}=0.3 \mathrm{~ns}$. The thresholds used in the figure are of the best performance chosen from a larger set of thresholds.

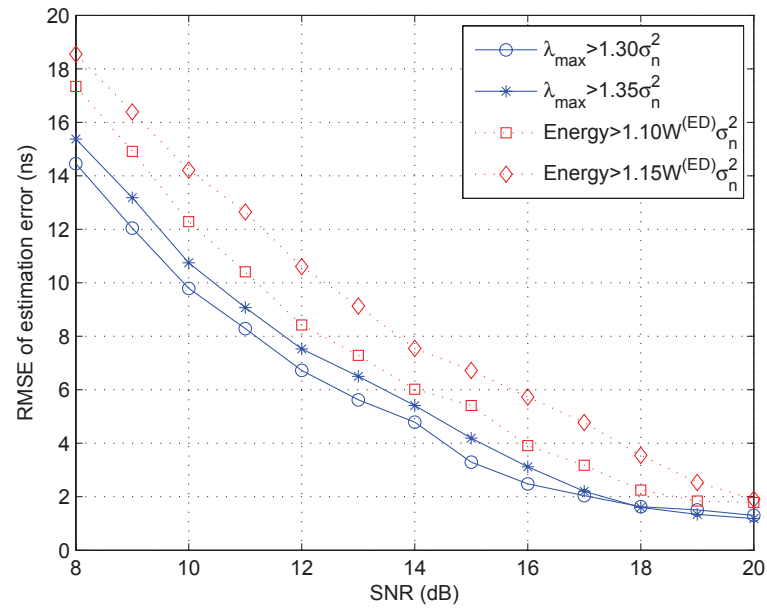

Fig. 4. Comparison of RMSE of ToA estimation errors of MED and ED, under 802.15.4a CM2 and $M=500$ trials. The other parameters for MED are $T_{s}=0.5 \mathrm{~ns}$ and $W^{(\mathrm{Eig})}=5$, and those for $\mathrm{ED}$ are $W^{(\mathrm{ED})}=3$ and $T_{b}=W^{(\mathrm{ED})} T_{s}=1.5 \mathrm{~ns}$.

in IEEE 802.15.3a and 802.15.4a channel models validate the higher accuracy of the max-eigenvalue method. Our new method thus represents an attractive alternative for low-complexity receivers in UWB ranging systems.

\section{REFERENCES}

[1] J. Foerster, "Channel modeling sub-committee report final," IEEE P802.1502/240-SG3a, 2004.

[2] A. F. Molisch, K. Balakrishnan, C. C. Chong, et al., "IEEE 802.15.4a channel model - final report," Sept. 2004. [Online]. Available: http://www.ieee802.org/15/pub/TG4a.htm

[3] V. Lottici, A. D'Andrea, and U. Mengali, "Channel estimation for ultrawideband communications," IEEE J. Select. Areas Commun., vol. 20, no. 9, pp. 1638-1645, Dec. 2002.

[4] J.-Y. Lee and R. A. Scholtz, "Ranging in a dense multipath environment using an UWB radio link," IEEE J. Select. Areas Commun., vol. 20, no. 9 , pp. 1677-1683, Dec. 2002.

[5] Y. Zeng, C. L. Koh, and Y.-C. Liang, "Maximum eigenvalue detection: theory and application," in International Conf. Communs. (ICC), pp. 4160 4164, May 2008

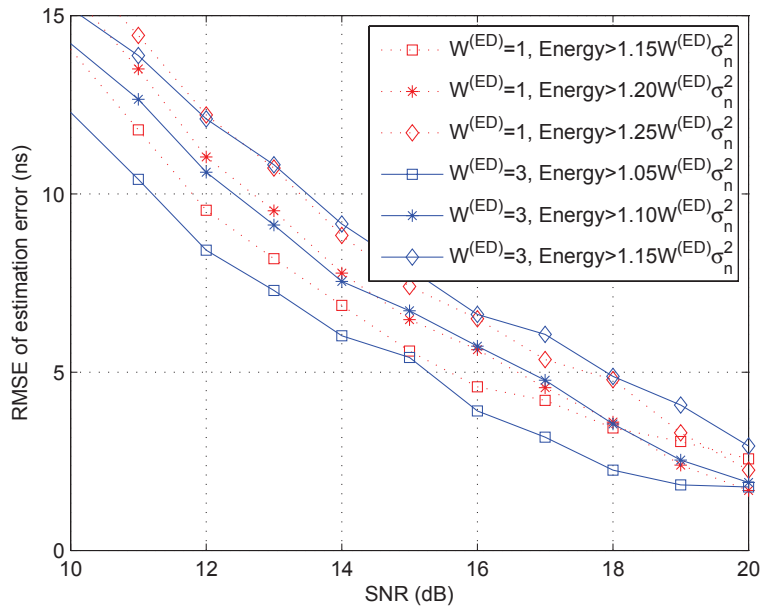

Fig. 5. Effect of the window size of ED on its ToA estimation accuracy. For $W^{(\mathrm{ED})}=1$ and $W^{(\mathrm{ED})}=3$, the minimum achievable RMSE minimized among all thresholds are distinguishable.

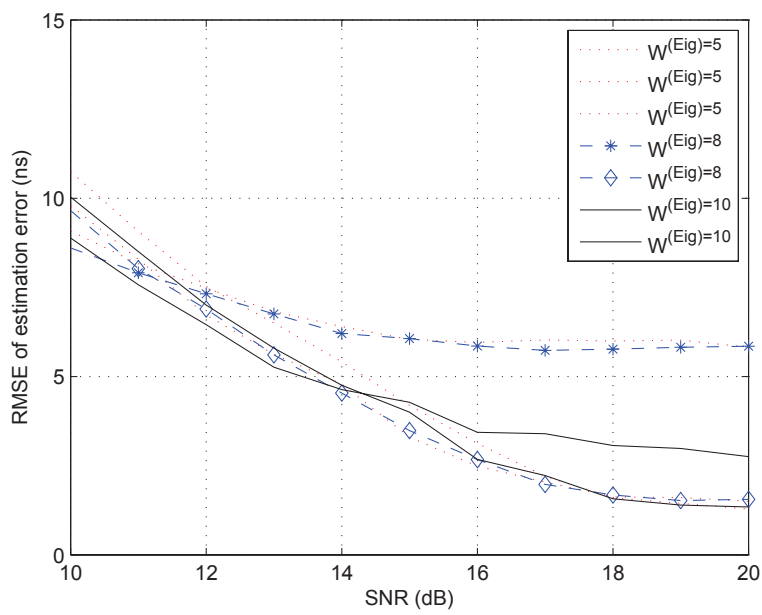

Fig. 6. Effect of the window size of MED on its ToA estimation accuracy. For $W^{(\mathrm{Eig})}=5, W^{(\mathrm{Eig})}=8$ and $W^{(\mathrm{Eig})}=10$, the curves of the minimum achievable RMSE minimized among all thresholds exhibits small difference.

[6] I. Guvenc, S. Gezici, and Z. Sahinoglu, "Ultra-wideband range estimation: theoretical limits and practical algorithms," in Proc. IEEE Int. Conf. on UWB (ICUWB), pp. 93-96, Sept. 2008.

[7] I. Guvenc, Z. Sahinoglu, P. Orlik, and H. Arslan, "Searchback algorithms for TOA estimation in non-coherent low-rate IR-UWB systems," Wireless Pers. Commun., vol. 48, no. 4, pp. 585-603, Mar. 2009.

[8] I. Guvenc and Z. Sahinoglu, "Threshold-based TOA estimation for impulse radio UWB systems," in Proc. Int. Conf. on Ultra-wideband (ICUWB), pp. 420-425, Sept. 2005.

[9] D. Dardari, C.C. Chong, and M. Z. Win, "Threshold-based time-of-arrival estimators in UWB dense multipath channels," in IEEE Trans. Commun., vol. 56, no. 8, pp. 1366-1378, Aug. 2008.

[10] A. Bejan, "Largest eigenvalues and sample covariance matrices. Tracy-Widom and Painleve II: computational aspects and realization in S-Plus with Applications," Preprint, available at www.cl.cam.ac.uk/aib29/TWinSplus.pdf.

[11] I. M. Johnstone, "On the distribution of the largest eigenvalue in principle components analysis," The Annals of Statistics, vol. 29, no. 2, 295-327, 2001.

[12] X. Li and K. Pahlavan, "Super-resolution TOA estimation with diversity for indoor geolocation," IEEE Trans. Wireless Commun., vol. 3, no. 1, pp. 224-234, Jan. 2004.

[13] A. F. Molisch, "Ultrawideband propagation channels: theory, measurement and models," IEEE Trans. Vehicular Technology, invited, no. 54, pp. 15281545, 2005.

[14] A. F. Molisch, Wireless Communications, 2nd ed., IEEE Press - Wiley, 2011. 